\title{
artigo
}

Azevedo, L.R.P.; Rodrigues, K.C.; Macedo, V.P.R.; Faria, C.A.

Perfil clínico-epidemiológico dos acidentes ofídicos ocorridos no Brasil

\section{Perfil clínico-epidemiológico dos acidentes ofídicos ocorridos no Brasil}

\author{
Clinical-epidemiological profile of snakebite accidents in Brazil \\ Perfil clínico-epidemiológico de los acidentes por mordeduras de serpiente em Brasil
}

\begin{abstract}
RESUMO
Objetivo. Analisar artigos científicos em periódicos on-line acerca dos aspectos epidemiológicos dos acidentes ofídicos no Brasil, para se obter melhores e mais precisas informações acerca do assunto. Método. Pesquisa descritiva delineada sobre uma revisão integrativa da literatura. Os passos foram: formulação da pergunta norteadora, busca de estudos em todas as bases de dados indexadas à BVS, SciELO e PubMed. Resultados. Foram localizados 105 artigos e 29 foram eleitos para coleta de dados. A maioria dos estudos foi na região Nordeste e Norte, durante o período de 2007 e 2011. Sobre as vítimas, a maior parte é do sexo masculino, reside em zonas rurais e está na faixa etária de 20 a 49 anos. Conclusão. 0 ofidismo ainda é um problema de saúde pública no Brasil que carece de estudos e ações voltadas ao tema a partir de seus fatores de risco e proteção associados, a fim de minimizar esse dano no cenário nacional.
\end{abstract}

DESCRITORES: Mordeduras de serpentes; epidemiologia; mordida de cobra; Serviços de saúde Pesquisa.

\section{ABSTRACT}

Objective. Analyze scientific articles in online journals about the epidemiological aspects of snakebites in Brazil, to obtain better and more accurate information on the subject. Method. Descriptive research outlined on an integrative literature review. The steps were: formulation of the guiding question, search for studies in all databases indexed to the BVS, SciELO and PubMed. Results. 105 articles were found and 29 were elected for data collection. Most of the studies were in the Northeast and North regions, during the period 2007 and 2011. About the victims, most of them are male, live in rural areas and are in the age group of 20 to 49 years. Conclusion. Ophidianism is still a public health problem in Brazil that lacks studies and actions focused on the topic based on its associated risk and protection factors, in order to minimize this damage in the national scenario.

DESCRIPTORS: Snake bites; epidemiology; snakebite; Health Services Research.

\section{RESUMEN}

Objetivo. Analizar artículos científicos en revistas en línea sobre los aspectos epidemiológicos de las mordeduras de serpientes en Brasil, para obtener información mejor y más precisa sobre el tema. Método Investigación descriptiva delineada en una revisión integradora de la literatura. Los pasos fueron: formulación de la pregunta orientadora, búsqueda de estudios en todas las bases de datos indexadas a la BVS, SciELO y PubMed. Resultados. Se encontraron 105 artículos y se eligieron 29 para la recolección de datos. La mayoría de los estudios se realizaron en las regiones Nordeste y Norte, durante el período 2007 y 2011. Sobre las víctimas, la mayoría son hombres, viven en áreas rurales y se encuentran en el grupo de edad de 20 a 49 años. Conclusión. El ofidianismo es todavía un problema de salud pública en Brasil que carece de estudios y acciones enfocadas en el tema con base en sus factores de riesgo y protección asociados, a fin de minimizar este daño en el escenario nacional.

DESCRIPTORES: Picaduras de serpiente; epidemiología; mordedura de serpiente; Investigación sobre servicios de salud.

RECEBIDO EM: 15/10/2020 APROVADO EM: 27/10/2020

\section{Larissa Rachel Príncipe Azevedo}

Acadêmicas do curso de graduação em Medicina da Universidade Federal do Amazonas - UFAM/ISB, Coari, Amazonas, Brazil. ORCID: 0000-0002-9418-5039

\section{Kerolaine da Cruz Rodrigues}

Acadêmicas do curso de graduação em Medicina da Universidade Federal do Amazonas - UFAM/ISB, Coari, Amazonas, Brazil. ORCID: 0000-0002-7098-002X 


\section{Valdenora Patrícia Rodrigues Macedo}

Docentes da Universidade Federal do Amazonas - UFAM/ISB, Coari, Amazonas, Brazil. ORCID: 0000-0002-8653-0883

\section{Carolina Arruda de Faria}

Docentes da Universidade Federal do Amazonas - UFAM/ISB, Coari, Amazonas, Brazil.

ORCID: 0000-0001-8086-1262

\section{INTRODUÇÃO}

A cidente ofídico ou ofidismo é o quadro de envenenamento decorrente da inoculação de uma peçonha e representa um problema de saúde pública que merece destaque por ocasionar grande morbimortalidade, sobretudo em países tropicais como o Brasil ${ }^{1}$. Em relação à epidemiologia, os envenenamentos por serpentes representam aproximadamente 125 mil óbitos anualmente no Brasil ${ }^{2}$. Importante destacar que estes números são subnotificados, uma vez que, no Brasil, ainda há regiões com dificuldade no acesso aos serviços de saúde, sobretudo na Amazônia. Logo, é de se esperar que haja dificuldade de acesso ao atendimento médico e, por conseguinte, ao registro de dados ${ }^{3}$.

As taxas de incidência e de morbimortalidade são marcantes, mas este dano à saúde ainda é reconhecido pela Organização Mundial da Saúde (OMS) como uma doença tropical negligenciada ${ }^{4,5}$. Afeta, em geral, populações de áreas remotas e rurais, favelas urbanas ou zonas de conflito. Como a maioria dos afetados tem pouca voz política, as doenças tropicais negligenciadas têm baixo impacto nas prioridades na agenda da saúde pública, o que causa além do pouco foco prático no problema, uma produção científica deficiente sobre o assunto, apesar de a questão possuir grande influência na saúde e qualidade de vida dos indivíduos ${ }^{4}$.

A partir da relevância do tema, este trabalho objetivou apresentar uma revisão integrativa da literatura que permitirá uma caracterização do perfil clínico e epidemiológico dos acidentes ofídicos ocorridos no Brasil durante o período 2009 a 2019. Com isso, esse trabalho contribuirá para averiguar as lacunas existentes sobre o assunto e assim contribuir com a comunidade científica.

\section{MÉTODO}

Trata-se de uma pesquisa descritiva com delineamento baseado em uma Revisão Integrativa da literatura. A partir da pergunta norteadora "Qual a produção científica existente no cenário nacional acerca da temática acidentes ofídicos e os aspectos epidemiológico no Brasil?", foi realizada uma busca de estudos em todas as bases de dados indexadas à BVS, SciELO e PubMed com os descritores: no idioma português: mordeduras de serpentes ou acidentes ofídicos; epidemiologia; em inglês: snakebite; Health Services Research; epidemiology. Os critérios de inclusão foram artigos disponíveis na integra e gratuitamente, nos idiomas português e inglês; publicados no período de 2009 a 2019; e foram excluídos trabalhos encontrados na modalidade tese, dissertação, editoriais e resumos. A pesquisa foi limitada por selecionar artigos envolvendo seres humanos. Para a melhor obtenção das informações dos artigos foi utilizado um instrumento para coleta de dados, conforme publicado por Souza, Silva e Carvalho (2010)6. (Anexo 1) Ademais, destaca-se que foi seguida a metodologia PRISMA $^{7}$ e o estudo foi realizado por duas pesquisadoras independentes.

\section{RESULTADOS}

Foram localizados 105 estudos. Depois de aplicados os critérios de inclusão e exclusão, foram excluídos artigos duplicados, os que não abordaram a temática ou apresentaram dados insuficientes para coleta. A partir disso, obteve-se 29 estudos finais. (Figura 1)

\section{ESTUDOS SELECIONADOS}

Cada artigo trouxe informações sobre os acidentes ofídicos em uma determinada região brasileira no período de 1945 a

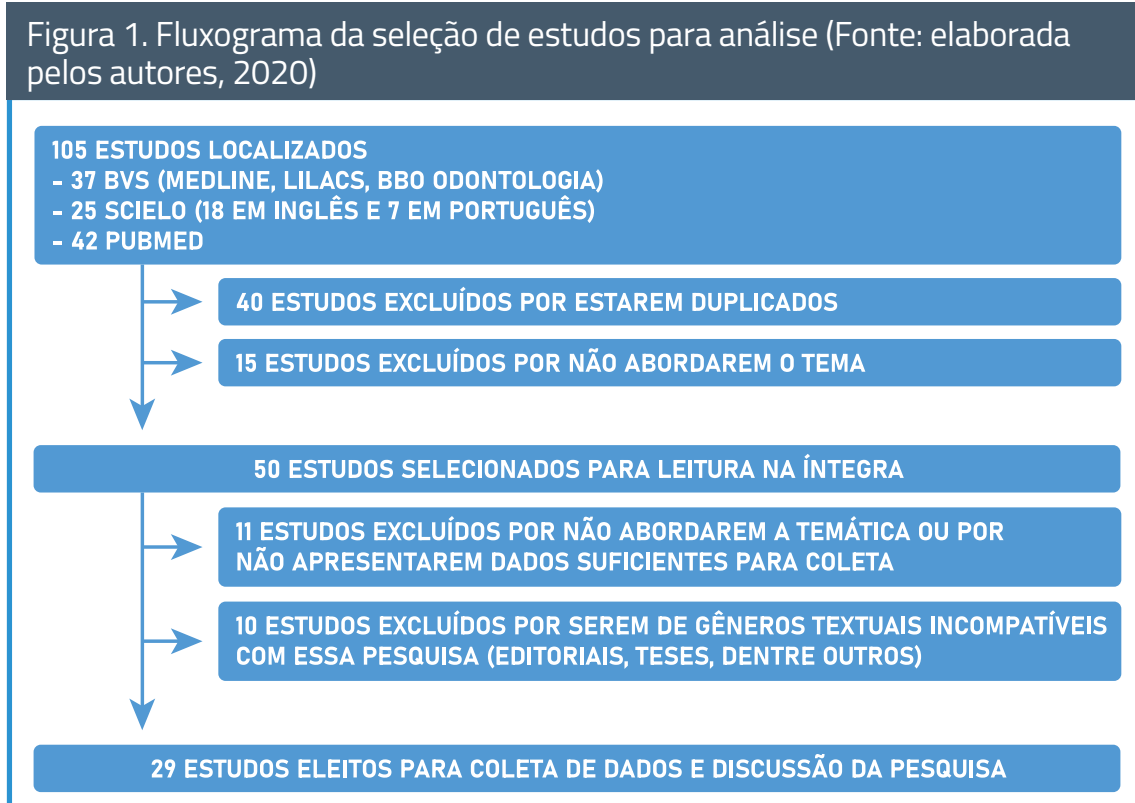


2019, uma vez que os estudos forneceram dados epidemiológicos e, portanto, apresentam informações de períodos anteriores à data de publicação. A maior parte dos artigos estudou o ano de 2010, com cerca de 20 artigos produzidos sobre esse período. Outros anos com grande foco de estudo sobre o tema foram 2007 a 2009 e 2011, com aproximadamente 15 estudos sobre cada ano. Ademais, a partir de tais dados, percebeu-se também uma queda constante na abordagem da temática a partir de 2014. (Gráfico 1)

Quanto às regiões brasileiras estudadas por cada artigo, as publicações científicas se deram, sobretudo, a respeito da região Nordeste, com $38 \%$ dos artigos e na região Norte, com 27\%, Na região Sul não houve nenhum estudo sobre o tema.

Com relação ao número de acidentes ofídicos relatados pelos estudos, houve um total de 125.098 casos, sendo a maior parte com vítimas do sexo masculino $(73,62 \%)$, enquanto vítimas do sexo feminino contaram com 23,8\%. Em 2,58\% dos casos relatados não houve classificação de sexo. Sobre a faixa etária mais prevalente das vítimas de ofidismo, não pode ser feita uma observação detalhada, visto que cada artigo utilizou uma referência diferente para abordar as idades de forma mais ampla. Porém, é possível observar uma tendência à prevalência de jovens adultos e adultos.

A maior parte dos estudos não referiu sobre a gravidade dos acidentes (76,71\%). Todavia, $13,93 \%$ dos casos foram relatados

Gráfico 1 - Período de dados epidemiológicos estudado pelos artigos (Fonte: elaborado pelos autores, 2020)

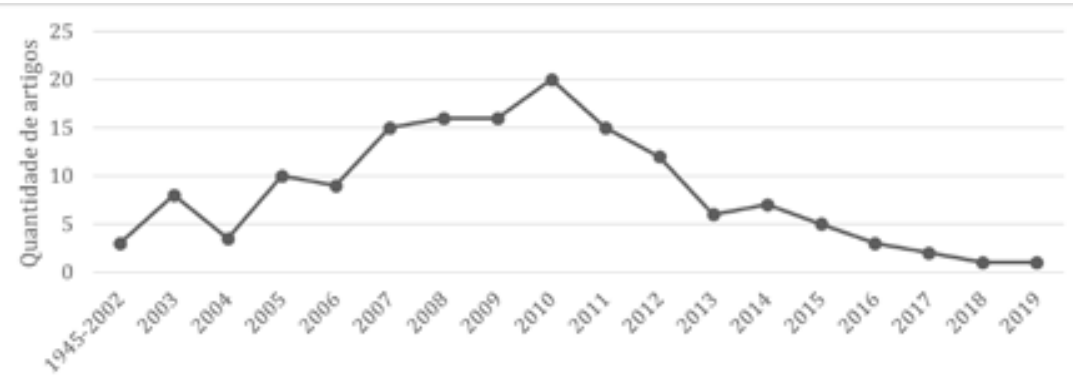

Período de tempo estudado pelos artigos
Sobre a evolução clínica, houve relato que $20,90 \%$ dos casos evoluíram com cura completa, já morte e evolução com sequelas ocorreram com menos de $1 \%$ dos casos em cada. Em 78,75\% dos casos a evolução clínica foi ignorada. Isolando tal parte ignora$\mathrm{da}$, tem-se que dos casos relatados, a grande maioria $(97,27 \%)$ obteve cura completa, $1,16 \%$ evoluiu com morte e $1,57 \%$ com sequelas. O perfil dos casos ainda apontou que a maior parte dos acidentes ocorreu em regiões rurais, com $60,77 \%$ dos casos. A zona urbana contou com $12,4 \%$ dos casos e a periurbana ou não-identificada, com $26,83 \%$. Outro dado coletado foi a respeito das serpentes: os acidentes ocorreram principalmente com gênero Bothrops, seguido de acidentes crotálicos e laquéticos. Muitos artigos não evidenciaram o gênero ou espécie, o que dificultou a sistematização das informações.

\section{DISCUSSÃO}

Os acidentes ofídicos representam um sério problema de saúde pública nos países tropicais, pela frequência com que ocorrem e pela morbimortalidade que causam. $\mathrm{Na}$ América do Sul, o Brasil é o país com maior número de acidentes com cerca de 20.000 casos por ano ${ }^{8}$. Sendo assim, a relevância deste estudo fica evidente.

A partir de 29 artigos selecionados conforme a metodologia especificada obteve-se relato de 125.098 casos de acidentes ofídicos entre o período de 2009 a 2019 no Brasil. A serpente majoritariamente descrita como causadora foi do gênero Bothrops, uma vez que está presente em todo o território nacional, seguida do gênero crotálico e laquético. Não foi descrita, na maior parte dos estudos, dados sobre espécies não-peçonhentas.

A maioria dos acidentes envolveu vítimas do sexo masculino e em idade ativa. A provável causa disso é o maior número de homens exercendo atividades que os expõem aos acidentes, como atividades extrativistas? Quanto à zona de ocorrência dos acidentes, foi descrita majoritariamente como rural. Todavia, houve grande destaque para regióes periurbanas ou não-identificadas. Quanto à zona rural, os dados obtidos foram confor- 
me esperado: são, naturalmente, as regiões em que há maior existência de serpentes em contato com humanos, pelas atividades exercidas em meios rurais. Já com relação ao destaque para regiões periurbanas ou não-identificadas, há duas hipóteses para isso: muitas regiões rurais não identificadas ou aumento na incidência de casos em regiões periurbanas, dado o processo de sinantropização desses animais por mudanças nos ecossistemas. Pode-se descrever como uma dessas mudanças o aumento na quantidade de resíduos domésticos produzidos e acondicionados precariamente nessas regiôes, onde estão grande parte dos bolsões de pobreza, que atraem roedores e seus principais predadores: as serpentes. Esta última hipótese demonstra que as cidades necessitam de maior organização, planejamento e infraestrutura, para evitar o processo de sinantropização, uma vez que traz prejuízos à população, como a ocorrência de acidentes ofídicos.

Os membros inferiores foram as regiões mais afetadas, seguidas de membros superiores, sendo esse padrão seguido por todos os artigos selecionados. Esse fato pode ser explicado por serem regiōes do corpo mais expostas e ativas, além de demonstrar a ausência de proteção individual adequada em muitos casos - o que poderia evitar tais acidentes ${ }^{10}$. No que se refere à gravidade dos acidentes, em grande parte dos estudos essa foi ignorada. Todavia, quando houve relato, a ocorrência foi maior de casos leves, seguido de moderados e graves. Apesar dos casos graves terem baixa ocorrência, é preciso ressaltar que há pacientes que demoram várias horas para procurar atendimento por dificuldades de acesso aos serviços de saúde, por exemplo, sobretudo em áreas remotas, o que prejudica o prognóstico das vítimas, principalmente nos casos mais graves ${ }^{11}$.

A evolução clínica das vítimas segue o padrão da gravidade dos acidentes: na maioria dos casos não houve relato, mas quando sim, teve destaque a cura completa e uma minoria evoluiu com morte ou cura com sequelas. A falta de dados e pesquisas sobre essa questão em específico é preocupante, visto que a análise da evolução é importante para avaliar de modo geral se o tratamento dos pacientes tem ocorrido de
Apesar dos casos graves terem baixa

ocorrência, é

preciso ressaltar

que há pacientes

que demoram várias

horas para procurar

atendimento por

dificuldades de

acesso aos serviços

de saúde, por

exemplo, sobretudo

em áreas remotas,

o que prejudica

o prognóstico

das vítimas,

principalmente nos

casos mais graves. forma eficiente e condiz com a gravidade esperada dos acidentes.

Orientando a discussão para os estudos selecionados, percebeu-se maior produção científica sobre as regiões Nordeste e Norte, uma vez que possuem maior incidência de casos. Importante evidenciar neste ponto ainda que essas regiões mais acometidas são também as economicamente menos equilibradas e desenvolvidas e o grupo de risco principal para o ofidismo são agricultores rurais empobrecidos, o que corrobora para menor investimento em pesquisas e ações sobre o tema. As populações rurais e/ou economicamente menos favorecidas geralmente têm pouca visibilidade e as vítimas têm dificuldade para influenciar os formuladores de políticas administrativas para atuarem quanto a esse dano à saúde. Como consequência disso, o ofidismo permanece na lista de Doenças Tropicais Negligenciadas da OMS, recebendo pouca atenção da comunidade global de saúde, indústria farmacêutica, dos governos e tendo baixa prioridade na agenda global de pesquisa em saúde ${ }^{12}$.

As outras regiões brasileiras tiveram menos destaque nas publicações, tendo, por exemplo, a região Sul com nenhum estudo. Entretanto, seria importante haver dados sobre a região, para averiguar se realmente não houve mudança no padrão de poucos casos durante o período investigado de dez anos.

A respeito dos anos estudados por cada artigo, percebeu-se grande número de publicações entre os anos de 2007 e 2011, com aproximadamente 15 artigos sobre cada ano, com pico no ano de 2010. A quantidade de estudos, porém, vêm diminuindo nos últimos anos, com queda crescente a partir de 2014, atingindo, por exemplo, a publicação de apenas um artigo no ano de 2018. Esse fato é bem preocupante, visto que esses acidentes ainda são um problema de saúde pública relevante, que merece atenção e necessita deixar de ser considerado negligenciado, devido ao seu impacto na qualidade de vida da população.

Uma limitação do presente estudo está relacionada à elevada proporção de variáveis ignoradas ou em branco, especialmente para aquelas que se referem ao gênero da serpente, à gravidade e evolução clínica dos 


\section{artigo}

Azevedo, L.R.P.; Rodrigues, K.C.; Macedo, V.P.R.; Faria, C.A.

Perfil clínico-epidemiológico dos acidentes ofídicos ocorridos no Brasil

casos e zona onde o acidente ocorreu, bem como tempo de atendimento e administração de soro. Essas informações são essenciais para se avaliar a necessidade de soro em cada região, orientar quais regiões merecem mais atenção e, quanto ao tempo de atendimento, é importante para verificar se as vítimas estão obtendo assistência com um período máximo adequado e também para investigar a implicação entre esse tempo e a evolução clínica e gravidade dos pacientes. Com a deficiência dessas informações, torna-se difícil a sistematização dos dados e a obtenção de discussões sobre o assunto.

Outro fator que se caracteriza como limitação do estudo é a ausência de pesquisas em determinadas áreas, sobretudo as mais remotas. Assim, a epidemiologia acaba por negligenciar aspectos dessas áreas e obter dados que podem não condizer com todas as realidades, o que dificulta ações em saúde específicas. Ademais, é essencial ressaltar que a subnotificação também influencia na interpretação do estudo. Se comparado com as regiôes Sul e Sudeste, o Nordeste ainda apresenta taxas de subnotificações maiores ${ }^{11}$.

A fim de sanar as lacunas evidenciadas, sugerem-se produções científicas que abordem melhor aspectos muitas vezes negligenciados em pesquisas, como administração de soro, tempo de atendimento e evolução clínica melhor especificada. O objetivo de tal sugestão é minimizar a grande proporção de variáveis ignoradas ou em branco, para melhorar a qualidade das informações e ampliar o conhecimento sobre o tema.

Sugere-se também maior quantidade de estudos, visto que a número de pesquisas vem diminuindo ao longo dos anos, o que dificulta a visibilidade sobre esse dano à saúde. Nesse sentido, inclusive, essencial seria a realização de mais pesquisas abordando áreas remotas, uma vez que são as regiões mais afetadas. Para esses estudos, sugere-se a utilização dos dados do Sistema de Informação de Agravos de Notificação,
Sinan, obtendo informações de qualidade, para que se possa traçar a real dimensão do problema nas diversas esferas de gestão do Sistema Único de Saúde ${ }^{11}$.

\section{CONCLUSÃO}

Os acidentes ofídicos são um problema de saúde pública no Brasil. Afeta principalmente homens em idade ativa, residentes de áreas rurais e periurbanas áreas e que provavelmente não utilizam equipamentos de proteção individual. A maior parte dos acidentes é causada por cobras do gênero Bothrops, com severidade moderada e evolução para cura completa. A produção científica sobre o tema vem diminuindo ao longo do tempo e, em sua maioria, aborda a região Nordeste do Brasil. Devido à morbimortalidade causada pelo ofidismo, altas taxas de incidência no cenário brasileiro e escassez de estudos nos últimos anos, faz-se necessário maior enfoque no tema em questão.

\section{REFERÊNCIAS}

1. Pinho F.M.O., Pereira I.D.. Ofidismo. Rev. Assoc. Med. Bras., 2001; 47( 1 ): 24-29.

2. Brasil. Ministério da Saúde. Acidentes de trabalho por animais peçonhentos entre trabalhadores do campo, floresta e águas, Brasil 2007 a 2017. Mar 2019; v.50, n.11.

3. Fiszon JT, Bochner, R. Subnotificação de acidentes por animais peçonhentos registrados pelo SINAN no Estado do Rio de Janeiro no período de 2001 a 2005. Rev. bras. Epidemiol, 2008; 11( 1 ): 114-127.

4. Tambourgi, DV. Envenenamento por serpentes: doença negligenciada afetando países em desenvolvimento. Anais da $62^{\circ}$ Reunião Anual da SBPC. Jul 2010; Natal, Brasil.

5. Lopes AB, Oliveira AA, Dias FCF, de Santana VMX, Oliveira VS, Liberato AA, Calado EJR, Lobo PHP, Gusmão KE, Guedes VR (2017) Perfil epidemiológico da coqueluche na região Norte do Brasil entre 2012 e 2015. Revista de Patologia do Tocantins, 4(2): 36-40.

6. Souza MT, Silva MD, Carvalho R. Revisão integrativa: o que é e como fazer. Einstein, 2010; 8:102-6.

7. Galvão TF, Pansani TSA, Harrad D. Principais itens para relatar Revisões sistemáticas e Meta-análises: A recomendação PRISMA. Epidemiol. Serv. Saúde, 2015; 24( 2 ): 335-342.

8. Lima JS, Martelli JH, Martelli DRB, Silva MS, Carvalho SFG, Canela JR et al . Perfil dos acidentes ofídicos no norte do Estado de Minas Gerais, Brasil. Rev. Soc. Bras. Med. Trop, 2009; 42( 5 ): 561-564.

9. Albuquerque PL, Silva GB Jr, Jacinto CN, Lima JB, Lima CB, Amaral YS, Veras Mdo S, Mota RM, Daher EF: Lesão renal aguda após acidente com picada de cobra atendido em centro terciário brasileiro. Nephrology, 2014; 19: 764-770.

10. Brasil. Ministério da Saúde. Sistema de Informação de Agravos de Notificação (SINAN). Casos de acidentes por serpentes. [Internet] Brasil, Grandes Regiões e Unidades Federadas, 2000 a 2013. Brasília, 2014. Available from: <http://www.saude.gov.br/ images/pdf/2014/julho/10/Tabela-06---CASOS---serpente--2000-a-2013---21-05-2014.pdf>. Acesso em: 24 de Outubro de 2020.

11. Saraiva, MG, Oliveira, DS, Filho, GMCS, Coutinho, LASA, Guerreiro, JV. Perfil epidemiológico dos acidentes ofídicos no Estado da Paraíba, Brasil, 2005 a 2010. Epidemiol. Serv. Saúde, 2012 ; 21( 3 ): 449-456.

12. LF Chaves, TW Chuang, M. Sasa, JM Gutierrez. As picadas de cobra estão associadas à pobreza, às flutuações do clima e ao El Niño. Sci Adv, 2015; p. e1500249.

13. Harrison, RA, Hargraves A, Wagstaff SC, Faragher B, Lalloo DG. Snake Envenoming: A Disease of Poverty. PLoS Negl Trop Dis 3(12): e569.

14. Lemos, JC, Almeida, TD, Fook, SML, Paiva, AA, Simões, MOS. 


\section{REFERÊNCIAS}

Epidemiologia dos acidentes ofídicos notificados pelo Centro de Assistência e Informação Toxicológica de Campina Grande (Ceatox-CG), Paraíba. Rev. bras. Epidemiol, 2009; 12(1): 50-59.

15. Saraiva, MG, Oliveira, DS, Filho Gilson Mauro Costa Fernandes, Coutinho Luiz Alberto Soares de Araújo, Guerreiro Jória Viana. Perfil epidemiológico dos acidentes ofídicos no Estado da Paraíba, Brasil, 2005 a 2010. Epidemiol. Serv. Saúde 2012; 21( 3 ): 449-456.

16. Leite RS, Targino ITG, Lopes YACF, Barros RM, Vieira AA. Epidemiologia dos acidentes com picadas de cobra nos municípios do estado da Paraíba, Brasil. Ciênc. saúde coletiva, 2013; 18 (5): 1463-1471.

17. Lima ACSF, Campos CEC, Ribeiro JR. Perfil epidemiológico de acidentes ofídicos do Estado do Amapá. Rev. Soc. Bras. Med. Trop., June 2009. Uberaba, v.42, n. 3, p. 329-335,

18. Magalhães SFV, , Samara Freire Valente et al. Snakebite envenomation in the Brazilian Amazon: a descriptive study. Transactions of The Royal Society of Tropical Medicine and Hygiene, v. 113, n. 3, p. 143-151, 2019

19. Oliveira FN, Brito MT, Morais ICOD, Fook SML, Albuquerque HND. Accidents caused by Bothrops and Bothropoides in the State of Paraiba: epidemiological and clinical aspects. Revista da Sociedade Brasileira de Medicina Tropical, 2010; 43(6):662-667.

20. Oliveira HFAD, Barros RM, Pasquino JA, Peixoto LR, Sousa $J A$, Leite R D S. Snakebite cases in the municipalities of the State of Paraíba, Brazil. Revista da Sociedade Brasileira de Medicina Tropical, 2013; 46(5):617-624.

21. Roriz KRPS, Zaqueo KD, Setubal SS, Katsuragawa TH, Silva RRD, Fernandes CFC, et all. Epidemiological study of snakebite cases in Brazilian Western Amazonia. Revista da Sociedade Brasileira de Medicina Tropical, 2018; 51(3):338-346.

22. Oliveira NDR, Sousa ACDR, Belmino JFB, Furtado SDS, Leite RDS. The epidemiology of envenomation via snakebite in the State of Piaui, Northeastern Brazil. Revista da Sociedade Brasileira de Medicina Tropical, 2015; 48(1):99-104.

23. Sangenis LHC, Lima DS, Leite GR, Calheiros VS, Rocha NDS, Nielebock MAP. Acidentes ofídicos no município de Valença, Rio de Janeiro: possível emergência de envenenamentos por cascavel. Revista de Patologia Tropical, 2013; 42(1):114-120.

24. Rita TS, Sisenando HA, Machado C. Análise epidemiológica dos acidentes ofídicos no município de Teresópolis-RJ no período de 2007 a 2010. Rev. Ciênc. Plural. 2016; 2(2):28-40.

25. Santos HLR, de Brito Sousa JD, Alcântara JA, Sachett JDAG, Boas TSV, Saraiva I, et all. Rattlesnakes bites in the Brazilian Amazon: Clinical epidemiology, spatial distribution and ecological determinants. Acta tropica, 2019; 191:69-76

26. Silva AMD, Mendes VKDG, Monteiro WM, Bernarde PS. Non-venomous snakebites in the Western Brazilian Amazon. Revista da Sociedade Brasileira de Medicina Tropical, 2019; 52: e20190120.

27. Tavares AV, Araújo KAMD, Marques MRDV, Vieira AA, Leite
RDS. The epidemiology of snakebite in the Rio Grande do Norte State, Northeastern Brazil. Revista do Instituto de Medicina Tropical de São Paulo, 2017; 59:e52.

28. Carmo ÉA, Nery AA, Jesus CSD, Casotti CA. Internações hospitalares por causas externas envolvendo contato com animais em um hospital geral do interior da Bahia, 2009-2011. Epidemiologia e Serviços de Saúde, 2016; 25:105-114

29. Costa MKBD, Fonseca CSD, Navoni JA, Freire EMX. Snakebite accidents in Rio Grande do Norte state, Brazil: Epidemiology, health management and influence of the environmental scenario. Tropical Medicine \& International Health, 2019; 24(4):432441.

30. De Medeiros CR, Hess PL, Nicoleti AF, Sueiro LR, Duarte MR, de Almeida-Santos SM, et all. Bites by the colubrid snake Philodryas patagoniensis: A clinical and epidemiological study of 297 cases. Toxicon, 2010; 56(6):1018-1024.

31. De Medeiros CR, de Souza SN, da Silva MC, de Souza Ventura J, de Oliveira Piorelli R, Puorto G. Bites by Tomodon dorsatus (serpentes, dipsadidae): Clinical and epidemiological study of 86 cases. Toxicon, 2019; 162:40-45.

32. Feitosa EL, Sampaio VS, Salinas JL, Queiroz AM, da Silva IM, Gomes AA, et all. Older age and time to medical assistance are associated with severity and mortality of snakebites in the Brazilian Amazon: A case-control study. PLoS one, 2015; 10(7):e0132237.

33. Albuquerque $\mathrm{PL}$, Silva Junior $\mathrm{GB}$, Jacinto $\mathrm{CN}$, Lima $\mathrm{CB}$, Lima JB, Veras MDSB, et al. Perfil epidemiológico dos acidentes por picada de cobra em região metropolitana do nordeste do Brasil. Revista do Instituto de Medicina Tropical de São Paulo, 2013; 55(5):347-351.

34. Alcântara JA, Bernarde PS, Sachett J, da Silva AM, Valente SF, Peixoto HM, et al. Stepping into a dangerous quagmire: Macroecological determinants of Bothrops envenomings, Brazilian Amazon. PloS one, 2018; 13(12):e0208532.

35. Alves EC, Sachett JAG, Sampaio VS, Souza JD, Oliveira SS, Nascimento EF, et al. Pedicting acute renal failure um Bothrops snabites patients in a tertiary reference center, Western Brazilian Amazon. PloS one, 2018; 13(8).

36. Aragon DC, Queiroz JAMD, Martinez EZ. Incidence of snakebites from 2007 to 2014 in the State of São Paulo, Southeast Brazil, using a Bayesian time series model. Revista da Sociedade Brasileira de Medicina Tropical, 2016; 49(4):515-519

37. Bernarde PS, Gomes JDO. Serpentes peçonhentas e ofidismo em Cruzeiro do Sul, Alto Juruá, estado do Acre, Brasil. Acta Amazonica, 2012; 42(1):65-72.

38. Bertolozzi MR, Scatena CMDC, França FODS. Vulnerabilities in snakebites in Sao Paulo, Brazil. Revista de saude publica, 2015; 49:82.

39. Bonan PRF, Lima JS, Martelli DRB, Silva MSD, Carvalho SFGD, Silveira MF, et al. Perfil epidemiológico dos acidentes causados por serpentes venenosas no norte do estado de Minas Gerais, Brasil. Revista Médica de Minas Gerais, 2010;20(4):503-507. 